\title{
A Convenient Nanosecond Laser Photolysis System for the Measurements of Transient Absorption Spectra on a Microsecond Time Scale
}

\author{
Toshihiro Nakayama*, Tetsuji Yamaguchi*, Kumao Hamanoue*, \\ Hiroshi Teranishi*, Toshihiko Nagamura**, Akio Mugishima**, \\ and Shinji SAKIMUKAI** \\ * Department of Chemistry, Kyoto Institute of Technology, \\ Matsugasaki, Sankyo-ku, Kyoto 606 \\ ** Unisoku Co. Ltd., Ohmine-Motomachi, Hirakata, Osaka 573-01
}

(Received November 10, 1986)

\author{
中山 敏弘* · 山口 哲司* . 演之上熊男* . 寺西 博*. \\ 長村 俊彦**·麦島 昭夫**. 崎向 伸二** \\ * 京都工芸㵶維大学工芸学部化学教室 京都市左京区松厅崎 （于606） \\ ** 株式会社ニニソク 大阪府枚方市大峰元町（广573-01） \\ （1986年11月10日受理）
}

\begin{abstract}
Synopsis
Combining a nanosecond ruby laser with an image sensor detector system and a microcomputer, a convenient laser photolysis system has been constructed and a transient absorption spectrum in a $400-\mathrm{nm}$ scanning region (at a delay time from $2 \mu \mathrm{s}$ to $1 \mathrm{~ms}$ ) can be recorded by one or two laser shots at a given temperature ranging from $88 \mathrm{~K}$ up to room temperature. The spectral resolution is $2.4 \mathrm{~nm}$, while the temporal resolution may be on the tenth of microseconds due to a limited pulse width of the probing light (FWHM=1.7 $\mu \mathrm{s}$ ). This laser photolysis system is convenient for discrimination of small differences in absorption spectra of short-lived species. Also, the system gives a correct absorption spectrum in the spectral region where strong emissions due to the transient species are observable.
\end{abstract}

\section{Introduction}

The transient absorption spectra of shortlived intermediates such as triplet states ${ }^{1,2)}$, free radicals $^{3)}$, radical ions ${ }^{4)}$ and complexes (exciplexes, charge-transfer complexes and ion pairs $)^{5,6)}$ are generally recorded by the separate measurements of the absorbances at a number of desired wavelengths, each following a single laser pulse, that is, a point-to-point illustration.
However, this method is not satisfactory enough for studying spectra which are the superposition of the absorptions due to more than two transient species. Neither is it suitable for the study of time-dependent spectral shifts, nor one can take the absorption spectra in the spectral region where strong fluorescences or phosphorescences are observable.

In the present paper, we report a somewhat simpler method of recording the absorption 


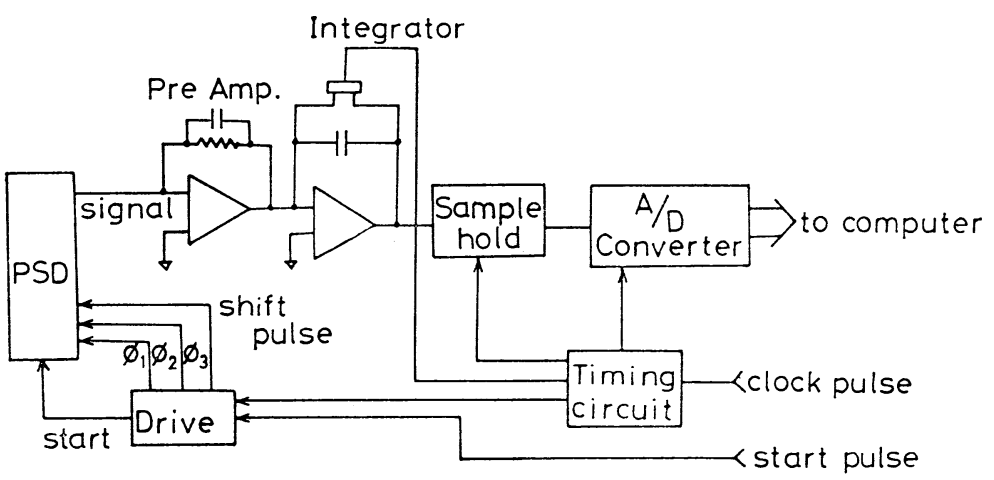

Fig. 1. Configuration of the ISD system.

spectra of short-lived transients generated by a single-laser pulse. Our present laser photolysis system is based on a combination of a nanosecond ruby laser with an image sensor detector system (ISD system) and a microcomputer.

\section{The ISD system and the recording of the absorption spectrum}

The configuration of the ISD system is shown in Fig. 1. A linear position sensitive detector (PSD) has the following characteristics: (1) A bipolar-type image sensor (HTV S2301-512Q) was used and the exposed part of each diode is large, i.e., $0.05 \times 2.5 \mathrm{~mm}$ square. (2) Since the capacitance of each diode is large, i.e., $46 \mathrm{pF}$, an external noise is satis- factorily eliminated.

A video signal from the PSD is amplified about 30 -fold by a preamplifier, and the video signals amplified are integrated by an integrator. In order to improve the precision of $A / D$ conversion, a sample hold circuit is coupled with the integrator. By these modifications, the sensitivity and the linearity of the detector system are increased more than five- and tenfolds, respectively, compared with those of a conventional MOS-type image sensor.

The experimental setup of our laser photolysis system is illustrated in Fig. 2. The photolysis is carried out by the second harmonic $(347.2 \mathrm{~nm})$ from a nanosecond ruby laser with a pulse width of $20 \mathrm{~ns}^{7}$. The optical path length of a cell is $10 \mathrm{~mm}$ and the sample solutions are degassed by several freeze-pump-

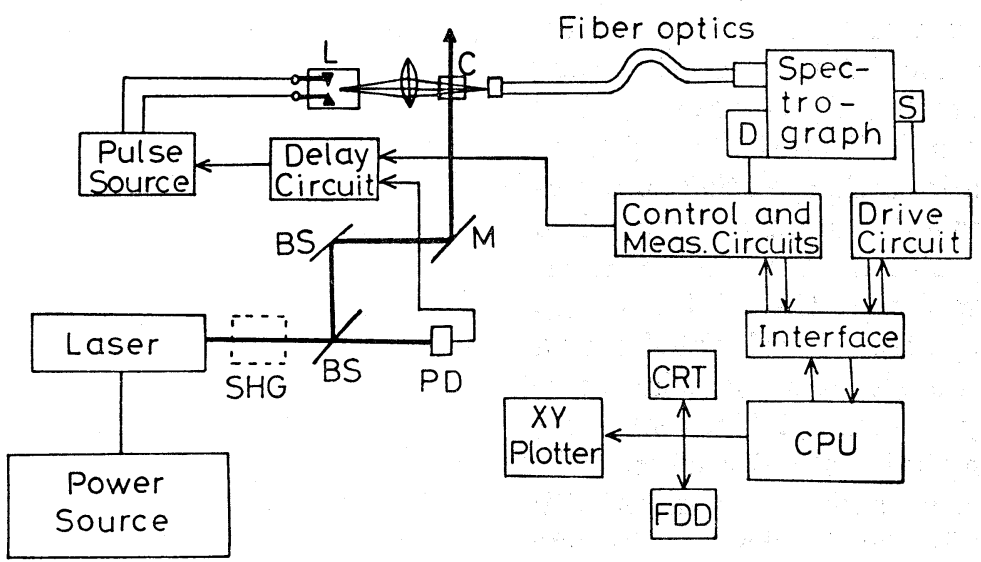

Fig. 2. Experimental setup of the laser photolysis system.

L; Flash lamp, C; Sample cell, D; Detector, S; Stepping motor, M; Mirror, BS; Beam splitter, $\mathrm{PD}$; Photo diode, SHG; Second harmonic generator. 


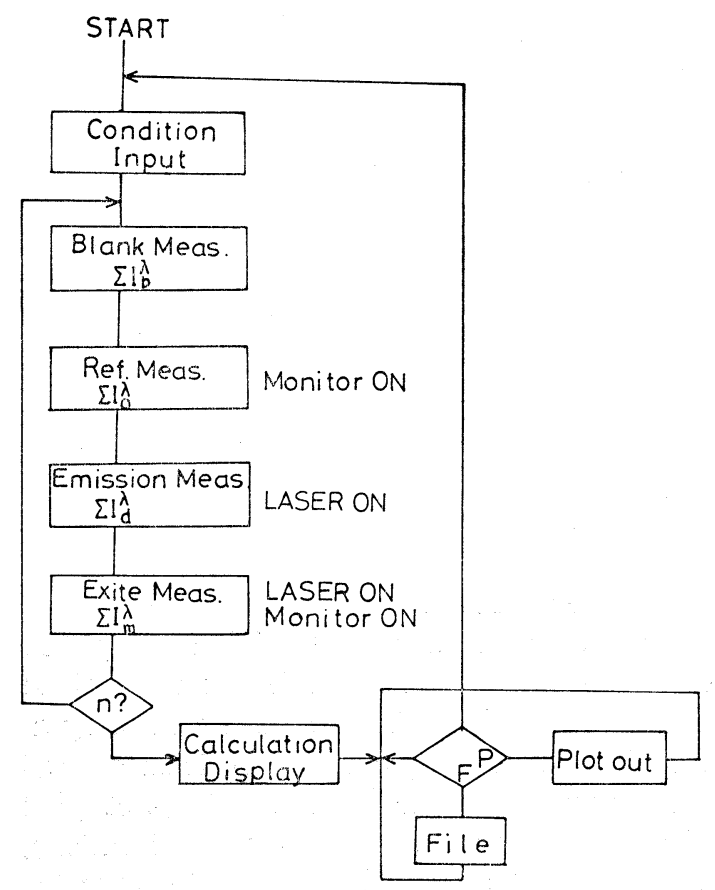

Fig. 3. Flow chart for the control of the laser photolysis system and data processing.

thaw cycles. The probing light from a pulsed xenon lamp (HTV L2435) passes through the cell and is imaged onto the entrance slit of a spectrograph (Unisoku M-200) by using a fiber optics (the entrance part is of $1.6 \mathrm{~mm}$ diameter and the exit part is of $0.4 \times 3.5 \mathrm{~mm}$ square): The full width at half-maximum intensity (FWHM) of the probing light is $1.7 \mu \mathrm{s}$.

The flow chart for the control of the laser photolysis system and data processing is shown in Fig. 3. After the intensity spectra (raw spectra) of the probing lights seen by PSD are transferred to a CPU (an NEC PC-9801Vm microcomputer), arithmetic operations are performed which convert the raw spectra into the absorbance spectrum. (The time constant for the data transfer to the CPU is $10 \mathrm{~ms}$ ). The absorbance spectrum is calculated over a range of 400-nm wavelengths by using three recorded intensity spectra ( $c f$. Fig. 4-a): One, $I_{0}^{\lambda}$, that is, probing-light intensity taken without the laser pulse; another, $I_{d}^{\lambda}$, taken with the laser pulse only, that is, the emission due to the excited species; the third, $I_{m}^{\lambda}$, taken with both the probing light and the laser pulse being fired. (When there is no emission,
$I_{d}^{2}$ is taken without the exciting laser pulse.) Thus, the absorbance spectrum, $A_{1}(t)$ at a delay time of $t$, is calculated by

$$
A_{1}(t)=\log \left[\left(I_{0}^{\lambda}-I_{b}^{\lambda}\right) /\left(I_{m}^{\lambda}-I_{d}^{\lambda}\right)\right]
$$

where $I_{b}^{\lambda}$ is the dark current spectrum of the detector system. The intensity and absorbance spectra are displayed on a CRT display and recorded by a X-Y plotter (a Nippon Denshikagaku Uniplotty UP-250) or stored on a flopy disk, as required.

In Fig. 4-a, we demonstrate the intensity spectra of $I_{0}^{\lambda}-I_{b}^{\lambda}, I_{d}^{\lambda}$ and $I_{m}^{\lambda}$ recorded without the exciting laser pulse. Thus, the absorbance spectrum, i.e., $A_{1}(t)$, corresponds to the base line spectrum, indicating that the base line drifts (or fluctuation of probing-light intensities) are very small ( $c f$. Fig. $4-\mathrm{b})$ : We have confirmed that the base line drifts are less than \pm 0.026 absorbance units. (Although $I_{m}^{2}$ in eq. (1) contains the dark current spectrum of the detector system as can be seen in Fig. 3 , it may be cancelled by taking the difierence

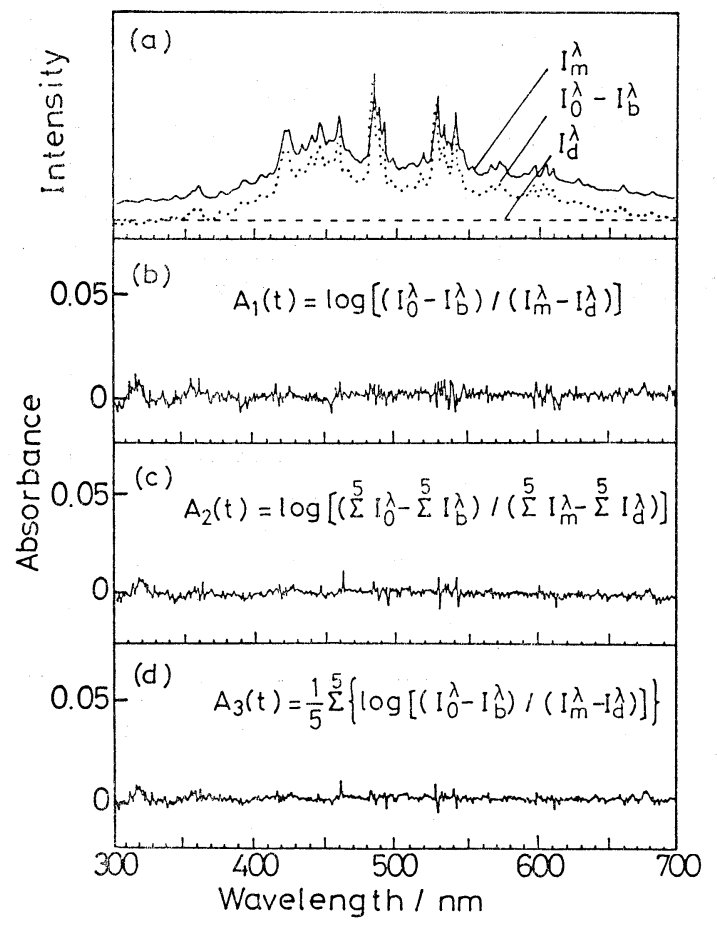

Fig. 4. Intensity spectra (a) taken without the exciting laser pulse and calibrated base line drifts (b, c, d). 
of $I_{m}^{\lambda}-I_{\hat{d}}^{\lambda}$. In fact, the base line drift is very small as can be seen in Fig. 4-b.)

Inevitably, the intensities of the probinglight and/or a laser pulse fluctuated slightly for the measurements of the $I_{0}^{2}, I_{d}^{\lambda}$ and $I_{m}^{2}$ spectra. Although such fluctuations were minimized by correct adjustment of the laser photolysis system, they led to some variability in the derived spectra. When improved signalto-noise ratios were required, these could be achieved by averaging a number of intensity spectra before deriving the absorption spectrum (cf. Fig. 3). In this case, the absorbance spectrum is calculated by

$$
A_{2}(t)=\log \left[\left(\sum^{n} I_{0}^{\lambda}-\sum^{n} I_{b}^{\lambda}\right) /\left(\sum^{n} I_{m}^{\lambda}-\sum^{n} I_{d}^{\lambda}\right)\right]
$$

or

$$
\left.A_{3}(t)=\frac{1}{n} \sum^{n}\left\{\log \left[I_{0}^{2}-I_{\hat{b}}^{2}\right) /\left(I_{m}^{2}-I_{d}^{2}\right)\right]\right\}
$$

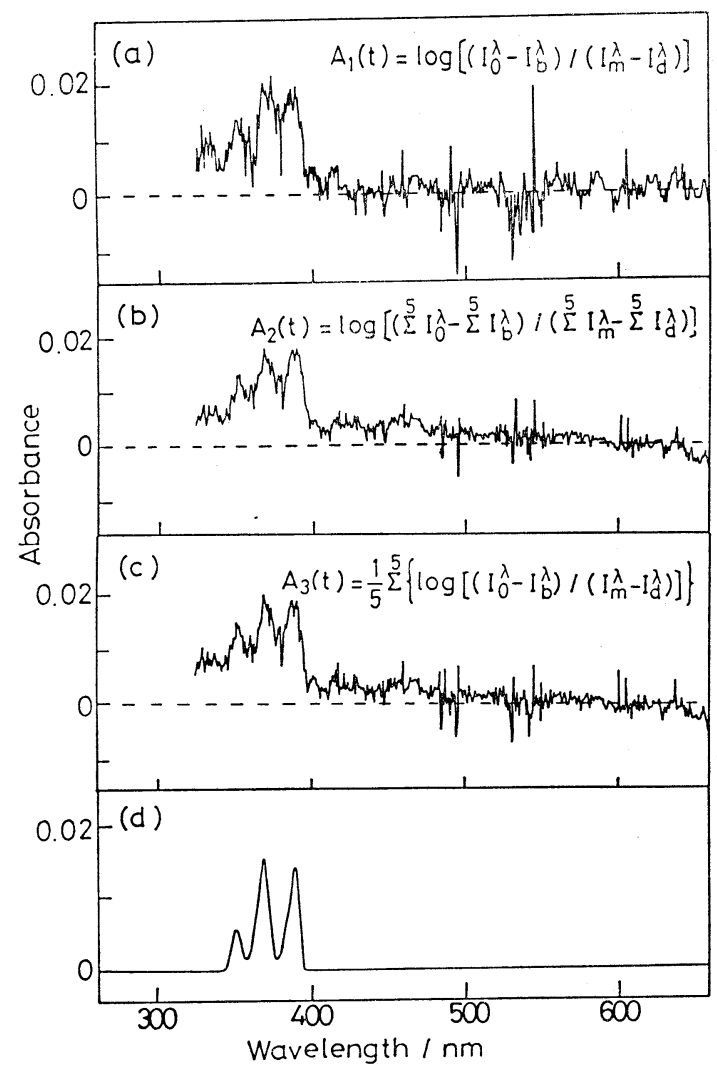

Fig. 5. Absorption spectra of 9-bromoanthracene $\left(2.19 \times 10^{-6} \mathrm{M}\right)$ in ethanol at room temperature, recorded by the ISD system $(\mathrm{a}, \mathrm{b}, \mathrm{c})$ and that recorded by a Hitachi 200-20 spectrophotometer (d). where $n$ is the number of averaging. The five-times averaged base line spectra derived from eqs. (2) and (3) are also shown in Figs. 4-c and d, respectively, indicating that both results give the base line drifts of less than \pm 0.006 absorbance units.

In Fig. 5, we show the absorption spectra of 9-bromoanthracene in ethanol $\left(2.19 \times 10^{-6} \mathrm{M}\right)$ derived from eqs. (1), (2) and (3), where the exciting laser pulse was cutted off. For the sake of comparison, we also show the absorption spectrum recorded using a Hitachi 200-20 spectrophotometer ( $c f$. Fig. 5-d), indicating that the relatively well-resolved spectra are obtained in Figs. 5-b and c: Although the absorption spectrum in Fig. 5-a is somewhat noisy, we confirmed that a well-resolved spectrum (derived by eq. (1)) similar to that in Fig. $5-\mathrm{d}$ was recorded for the concentration of 9-bromoanthracene to be $\sim 1.75 \times 10^{-5} \mathrm{M}$ (the maximum absorbance $\approx 0.12$ ).

When an absorption spectrum is recorded at $77 \mathrm{~K}$ in liquid nitrogen, the spectrum sometimes exhibited a base line drift due to the bubbling of liquid nitrogen. Thus, we have constructed a special cell holder as shown in Fig. 6, and an absorption spectrum at a given temperature ranging from $88 \mathrm{~K}$ up to room temperature could be easily recorded within a controlled temperature of $\pm 0.5^{\circ} \mathrm{C}$.

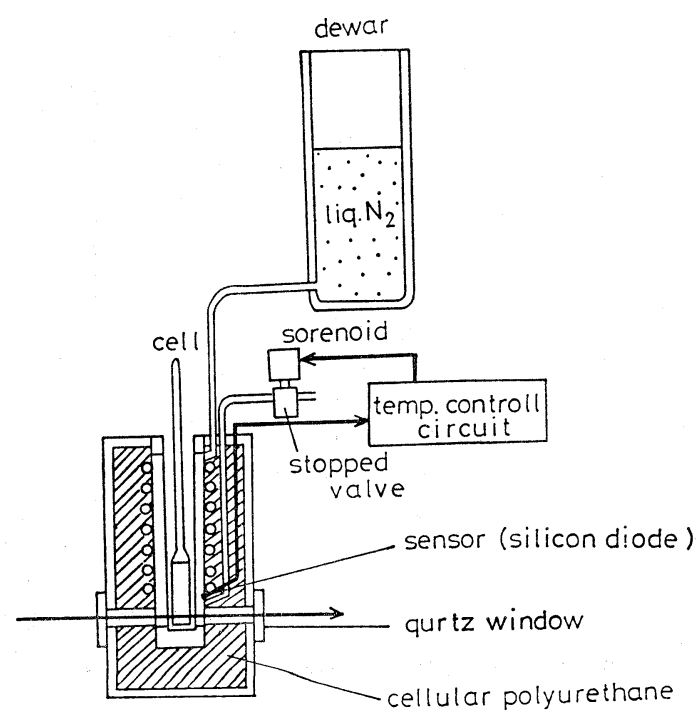

Fig. 6. Schematic diagram of the temperaturecontrolled cell holder. 


\section{Resolution and linearity}

The spectrograph is a crossed Czerny-Turner type with collimating and focussing mirrors of focal length $200 \mathrm{~mm}$ and an overall focal ratio of $\mathrm{f} / 3.5$, and has the following characteristics of groves, blaze and dispersion, respectively: 300 lines $/ \mathrm{mm}, \quad 500 \mathrm{~nm}, \quad 15.6 \mathrm{~nm} / \mathrm{mm}$. The entrance slit of the spectrograph is $10 \mathrm{~mm}$ high (a width being adjustable between 0.05 and $2 \mathrm{~mm}$ ) and the exit part is directly connected to the exposed part of the PSD $(2.5 \mathrm{~mm}$ high and $25.6 \mathrm{~mm}$ width). With the focal plane of the focus mirror coincident with the PSD, the above grating is able to span approximately $400 \mathrm{~nm}$. The particular wavelength range spanned can be adjusted by simply turning the grating by a stepping motor controlled by the CPU.

When the probing light is perfectly focussed onto the entrance slit of the spectrograph, the dispersed light at the exit focal plane should have a wavelength distribution that is linear with distance across the focal plane. Thus, the wavelength associated with each channel of the PSD would be a linear function of the channel number. In practice, this ideal situation is not exactly realized: In our laser photolysis system, the probing light is not focussed onto the entrance slit of the spectrograph, that is, a fiber optics $(3.5 \mathrm{~mm}$ high and $0.4 \mathrm{~mm}$ width) is not directly connected to the entrance slit of the spectrograph and some misfocussing is unavoidable. Thus, the wavelength scale associated with record spectra is not strictly linear; however, we have confirmed that the spectral shift between two spectra recorded by the ISD system and a Hitachi 200-20 spectrophotometer is about $1 \mathrm{~nm}$ at the outer sides of the absorbance spectra.

The resolution of our laser photolysis system was tested by the measurement of the light intensities from a $\mathrm{He}-\mathrm{Ne}$ laser and a $\mathrm{Hg}$-lamp as the line sources. The line width (FWHM) was checked for various entrance slit widths, and the results are shown in Fig. 7 and Table 1 ; in Fig. 7, full and dotted lines are the emission spectra recorded at the slit widths of 0.05 and $2 \mathrm{~mm}$, respectively. Also, the absorption spectra of a holmium glass filter (Hoya HY-1) at various entrance slit widths were recorded without the exciting laser

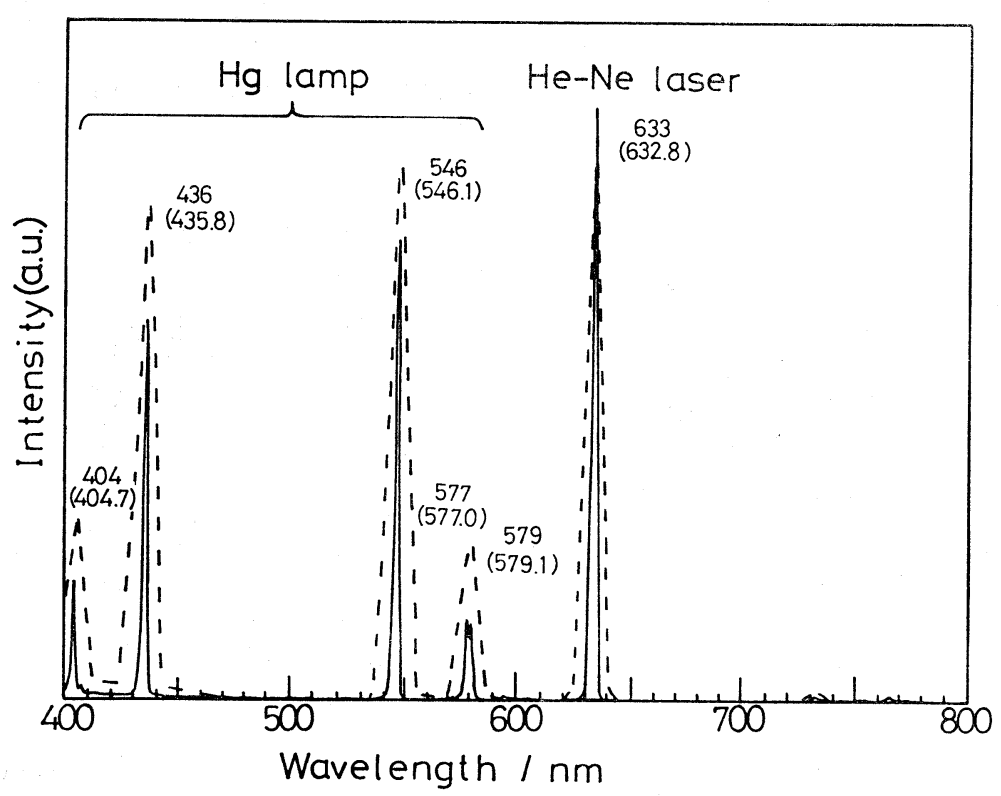

Fig. 7. Intensity spectra of the lights from a He-Ne laser and a Hg-lamp, recorded by the ISD system at the slit widths of 0.05 (full line) and $2.0 \mathrm{~mm}$ (broken line). The numbers are the line-wavelengths read actually and those in parentheses are wellknown line-wavelengths. 
Table I. The full width at half-maximum intensities (FWHM) of the lights from a He-Ne laser and a Hg-lamp measured by the ISD system at various slit widths.

\begin{tabular}{cccc}
\hline & \multicolumn{3}{c}{ FWHM/nm } \\
\cline { 2 - 4 } $\begin{array}{c}\text { Slit } \\
\text { width } \\
\text { /mm }\end{array}$ & \begin{tabular}{c} 
He-Ne \\
laser \\
at \\
\cline { 2 - 4 }
\end{tabular} & \multicolumn{2}{c}{ Hg-lamp } \\
\cline { 3 - 4 } $032.8 \mathrm{~nm}$ & $436 \mathrm{~nm}$ & $\begin{array}{c}\text { at } \\
546.4 \mathrm{~nm}\end{array}$ \\
\hline 0.05 & 2.0 & 2.0 & 2.0 \\
0.1 & 2.4 & 2.4 & 2.2 \\
0.2 & 2.8 & 2.8 & 2.8 \\
0.5 & 5.6 & 5.6 & 5.8 \\
1.0 & 7.4 & 7.6 & 7,5 \\
2.0 & 7.4 & 7.8 & 7.5 \\
\hline
\end{tabular}

pulse. Typical examples for the slit widths of 0.05 and $2 \mathrm{~mm}$ are shown by full and dotted lines, respectively, in Fig. 8-a. (A spectrum shown in Fig. 8-b is the absorption spectrum recorded by a Hitachi 200-20 spectrophotometer of $2.5 \mathrm{~nm}$ resolution.) In Table II, the relative intensities of the absorption peaks of a Hoya HY-1 are listed. All the results obtained so far indicate that a slit width narrower than $0.1 \mathrm{~mm}$ gives a correct spectrum with spectral resolution of $2.4 \mathrm{~nm}$.

The linearity of readout (the absorbance value) was tested using neutral density filters with nominal absorbances of $0.15,0.30,0.60$ and 1.0 which were individually calibrated over a range of wavelengths using a Hitachi 200-20 spectrophotometer. The results obtained at 400, 500, 600 and $700 \mathrm{~nm}$ are shown in Fig. 9-a. We also show the results for 9-

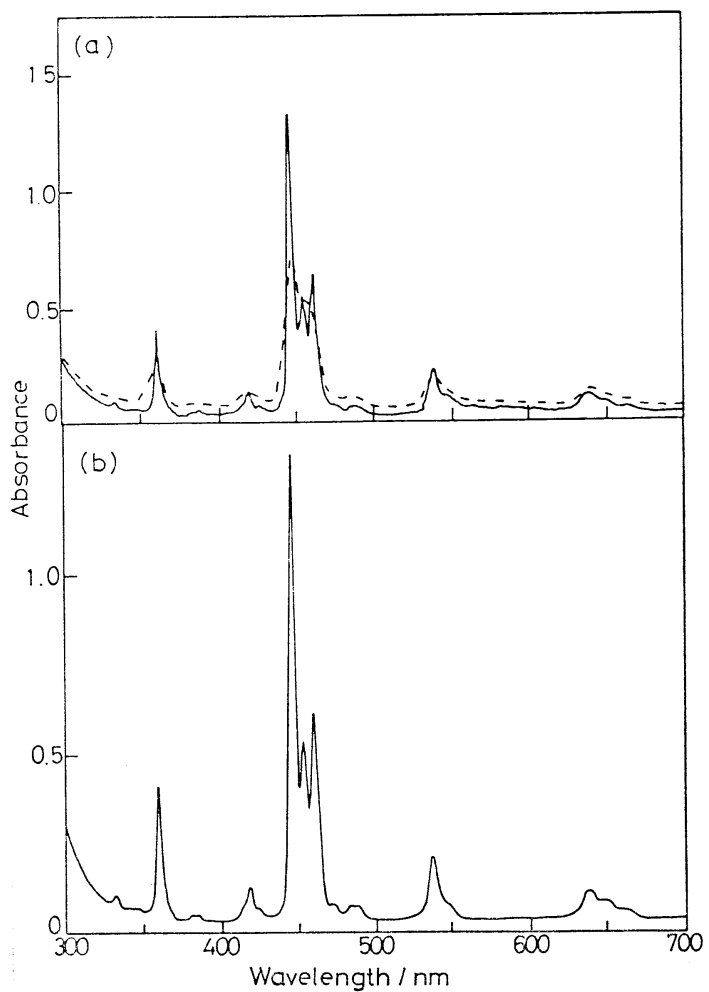

Fig. 8. Absorption spectra of a holmium glass filter recorded by the ISD system (a) and a Hitachi 200-20 spectrophotometer (b). Slit widths for the spectra shown by full and dotted lines in (a) are 0.05 and 2.0 $\mathrm{mm}$, respectively.

bromoanthracene in ethanol ( $c f$. Fig. 9-b), where the absorbance values were measured at $350.5,369.5$ and $390.0 \mathrm{~nm}$. (In both cases, the exciting laser pulse was cutted off.) All

Table II. Comparison of the relative intensities of the absorption peaks of a Hoya HY-1 recorded by ISD system with that by a Hitachi 200-20 spectrophotometer.

\begin{tabular}{|c|c|c|c|c|c|}
\hline \multirow{2}{*}{ Slit width $/ \mathrm{mm}$} & \multicolumn{5}{|c|}{$I\left(\lambda_{\max }\right) / I\left(\lambda_{\max }=446 \mathrm{~nm}\right)$} \\
\hline & at $361 \mathrm{~nm}$ & at $446 \mathrm{~nm}$ & at $454 \mathrm{~nm}$ & at $460.5 \mathrm{~nm}$ & at $537.5 \mathrm{~nm}$ \\
\hline 0.05 & 0.31 & 1 & 0.40 & 0.46 & 0.16 \\
\hline 0.1 & 0.31 & 1 & 0.40 & 0.46 & 0.16 \\
\hline 0.2 & 0.33 & 1 & 0.46 & 0.50 & 0.18 \\
\hline 0.5 & 0.33 & 1 & 0.52 & 0.58 & 0.22 \\
\hline 1.0 & 0.35 & 1 & \multicolumn{2}{|c|}{$0.66^{\mathrm{a})}$} & 0.24 \\
\hline 2.0 & 0.37 & 1 & \multicolumn{2}{|c|}{$0.70^{\mathrm{a})}$} & 0.26 \\
\hline Hitachi $200-20$ & 0.31 & 1 & 0.40 & 0.46 & 0.16 \\
\hline
\end{tabular}

a) Two peaks at 454 and $460.5 \mathrm{~nm}$ could not be well-resolved. 


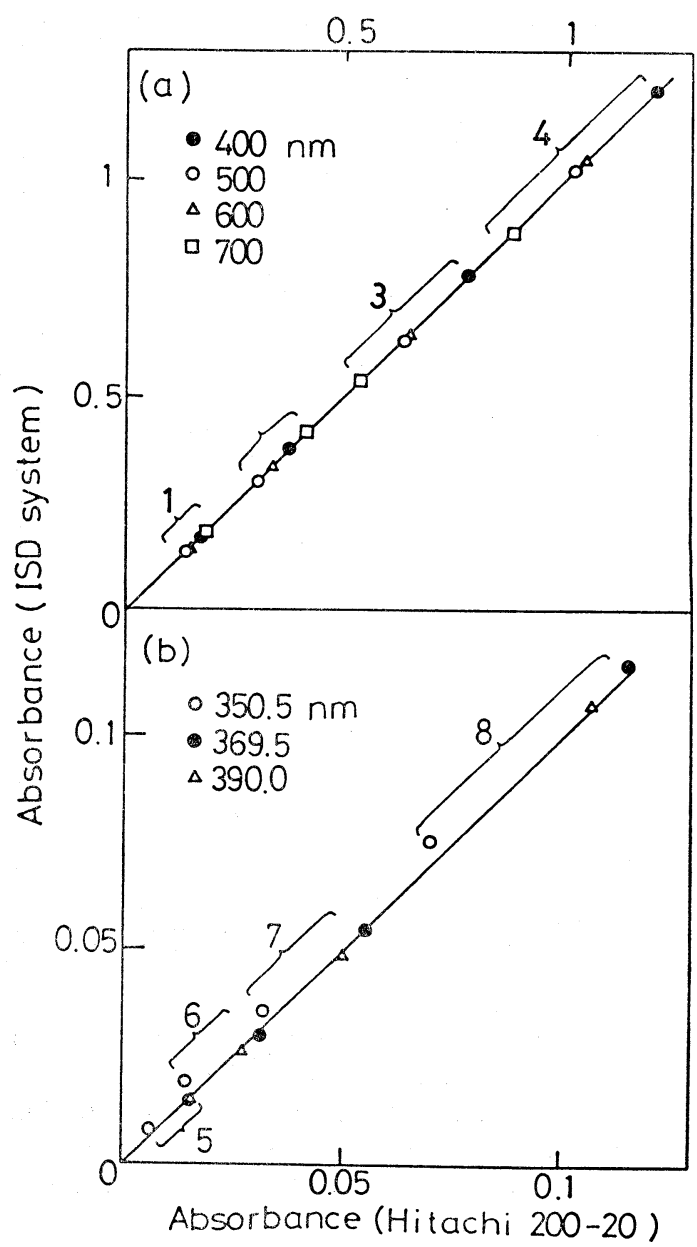

the values lie on a straight line with the zerointercept and the slope of unity, respectively, indicating that absorbance values measured by the ISD system are reasonably accurate and the response is essentially linear.

\section{Transient absorption spectral measurements}

The ISD system was used to record the triplettriplet $\left(T^{\prime} \leftarrow T_{1}\right)$ absorption of the lowest triplet state $\left(T_{1}\right)$ of anthraquinone (AQ) in EPA (ether/ isopentane/ethanol $=5: 5: 2$ in volume ratio) at $88 \mathrm{~K}$ ( $c f$. Fig. 10). The slit width of the spectrograph was $0.1 \mathrm{~mm}$. The absorption spectrum (full line in Fig. 10) recorded by the ISD system is identical with that recorded by a conventional system (open circles) in EPA at $77 \mathrm{~K}^{2}$. On account of the strong phosphorescence emission of $\mathrm{AQ}$, we could not observe

Fig. 9. Comparison of the absorbance values measured by the ISD system with those measured by a Hitachi 200-20 spectrophotometer, where neutral density filters (a) and 9-bromoanthracene in ethanol (b) were used for the measurements of the absorbances. The nominal absorbances of the neutral density filters are $0.15(1)$, 0.30 (2), 0.60 (3) and 1.0 (4), and the concentrations of 9 -bromoanthracene in ethanol are $2.19 \times 10^{-6}(5), 4.38 \times 10^{-6}(6)$, $8.75 \times 10^{-6}(7)$ and $1.75 \times 10^{-5} \mathrm{M}(8)$.

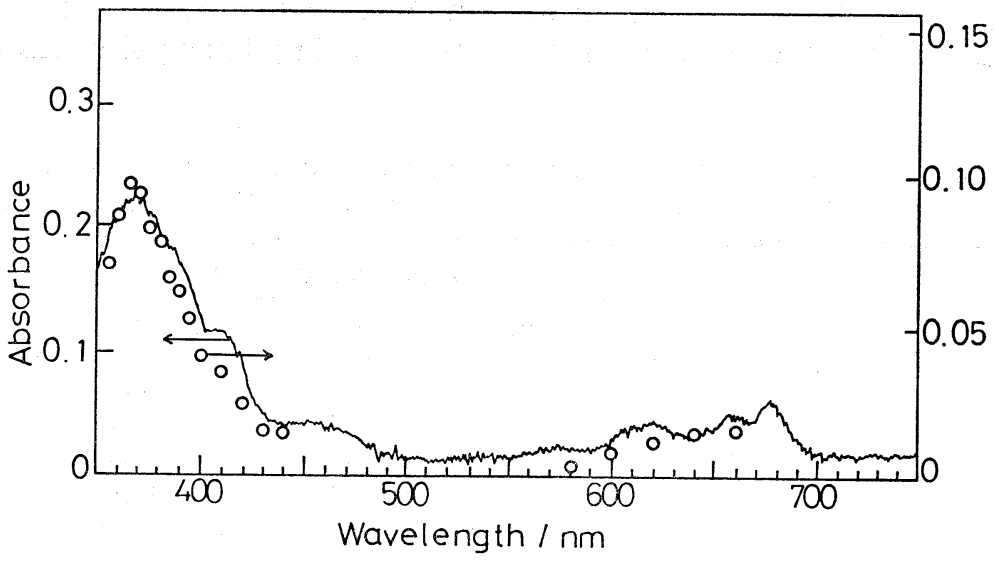

Fig. 10. The $T^{\prime} \leftarrow T_{1}$ absorption spectrum of $\mathrm{AQ}$ recorded by the ISD system at $88 \mathrm{~K}$ in EPA (full line) and that recorded by a conventional system at $77 \mathrm{~K}$ in EPA (open circles)2. 


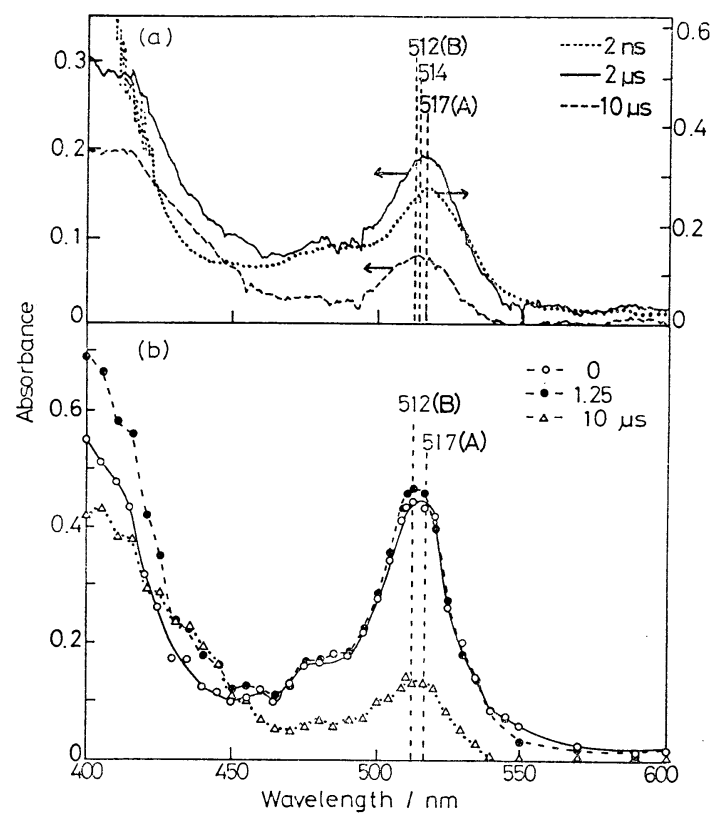

Fig. 11. Transient absorption spectra recorded by the ISD system at the delay times of 2 (full line) and $10 \mu$ s (broken line) upon the nanosecond laser photolysis of $\mathrm{AQ} / \mathrm{TEA}(0.2 \mathrm{M})$ in toluene at room temperature. The spectrum shown by a dotted line in (a) and those in (b) are our previous results obtained upon the pico- and nanosecond laser photolyses, respectively5).

any absorptions at 450 to $570 \mathrm{~nm}$ by a conventional system, while the ISD system gives rise to a clear absorption spectrum.

Figure 11-a demonstrates the transient absorption spectra recorded by the ISD method at the delay times of 2 (full line) and $10 \mu \mathrm{s}$ (broken line) upon the nanosecond laser photolysis of $\mathrm{AQ} / \mathrm{TEA}(0.2 \mathrm{M})$ in toluene at room temperature. In the previous paper ${ }^{5)}$, we reported that: (1) The picosecond laser photolysis of AQ/triethylamine (TEA, $0.2 \mathrm{M}$ ) in toluene ( $c f$. dotted line in Fig. 11-a) or ethanol gives rise to the appearance of the absorption spectrum with Band A similar to that of radical anion of AQ. (2) Nanosecond laser photolysis revealed that Band A appeared within the duration of the laser pulse and then Band A shifted to Band $\mathrm{B}$ by $\approx 5 \mathrm{~nm}$ in toluene ( $c f$. Fig. $11-b$ ) and $\approx 10 \mathrm{~nm}$ in ethanol. (3) Band $B$ decayed following a first-order reaction kinetics. Moreover, no photocurrents due to Bands $\mathrm{A}$ and $\mathrm{B}$ were observed.

Thus, we assigned Bands $\mathrm{A}$ and $\mathrm{B}$ to an exciplex and a contact ion pair, respectively, between $\mathrm{AQ}$ and TEA. Clearly, one can see that the spectra recorded by the ISD system are consistent with those recorded by a conventional system. Strictly speaking, however, the spectrum at a delay time of $2 \mu$ s in Fig. 11-a should have the absorption maximum at $512 \mathrm{~nm}$. The appearance of the absorption maximum at $514 \mathrm{~nm}$ may be due to the fact that the pulse width of the probing light is $1.7 \mu \mathrm{s}$ (FWHM).

\section{Conclusion}

We have presented a convenient method for the measurements of the transient absorption spectrum by the nanosecond laser photolysis: We call the present ISD system coupled with a microcomputer a Unisoku USP-500 system, hereafter. Since the integrated probing-light intensity seen by the PSD is proportional to the duration of the probing-light pulse (FWHM $=1.7 \mu \mathrm{s}$ ), the time-resolved absorption spectrum of the temporal resolution on the tenth of microseconds may satisfactorily be discussed. A shorter probing pulse, giving a corresponding improvement in the temporal resolution, could be used, but, it is limited with the technical difficulty. An alternative method is to use the gated ISD system, but this would require a probing-light pulse that is more intense than could be achieved in the present system. Such a high intensity of the probing light may cause some photochemical reactions of the samples in high yields. Thus, the most desirable way may be to use a gated ISD system with an intensified image converter. Technical test along this line is in progress; The preliminary result was presented elsewhere ${ }^{8)}$.

\section{References}

1) K. Hamanoue, T. Nakayama, T. Miyake, and H. Teranishi: Chem. Lett. 39 (1981); K. Hamanoue, K. Yokoyama, Y. Kajiwara, K. Nakajima, T. Nakayama, and H. Teranishi: Chem. Phys. Let. 110, 25 (1984); K. Hamanoue, M. Amano, M. Kimoto, Y. Kajiwara, T. Nakayama, and 
H. Teranishi: J. Am. Chem. Soc. 106, 5993 (1984); T. Nakayama, T. Kuramoto, K. Hamanoue, and H. Teranishi: J. Phys. Chem. 90, 5689 (1986).

2) K. Hamanoue, Y. Kajiwara, T. Miyake, T. Nakayama, S. Hirase, and H. Teranishi : Chem. Phys. Lett. 94, 276 (1983).

3) T. Nakayama, K. Hamanoue, T. Hidaka, M. Okamoto, and H. Teranishi: J. Photochem. 24, 71 (1984); K. Hamanoue, T. Nakayama, A. Tanaka, Y. Kajiwara, and H. Teranishi: J. Photochem. 34, 73 (1986).

4) K. Hamanoue, H. Teranishi, A. Sugawara, S. Tagawa, and Y. Tabata: Mem. Fac. Ind. Arts, Kyoto Tech. Univ., Sci. Technol. 27, 86 (1978); K. Hamanoue, S. Tai, T. Hidaka, T. Nakayama, M. Kimoto, and H. Teranishi: J. Phys. Chem. 88, 4380 (1984); K. Hamanoue, M. Kimoto, T. Nakayama, H. Teranishi, S. Tagawa, and Y. Tabata: Radiat. Phys. Chem. 24, 445 (1984);
T. Nakayama, M. Kimoto, K. Hamanoue, H. Teranishi, M. Washio, S. Tagawa, and Y. Tabata: Radiat. Phys. Chem. in press.

5) K. Hamanoue, K. Yokoyama, Y. Kajiwara, M. Kimoto, T. Nakayama, and H. Teranishi: Chem. Phys. Lett. 113, 207 (1985).

6) K. Hamanoue, T. Nakayama, K. Sugiura, H. Teranishi, M. Washio, S. Tagawa, and Y. Tabata: Chem. Phys. Lett. 118, 503 (1985); K. Hamanoue, M. Kimoto, Y. Kajiwara, T. Nakayama, and H. Teranishi: J. Photochem. 31, 143 (1985).

7) T. Nakayama, T. Miyake, M. Okamoto, K. Hamanoue, and H. Teranishi: Mem. Fac. Ind. Arts, Kyoto Tech. Univ., Sci. Technol. 29, 35 (1980).

8) T. Yamaguchi, T. Nakayama, K. Hamanoue, H. Teranishi, and T. Nagamura: Symposium on Photochemistry, p. 45, Sakai Japan (1986). 